\title{
Backsplash studies for the SPD subdetector of LHCb
}

\section{Míriam Calvo Gómez*}

University of Barcelona, Spain

E-mail: miriam@ecm.ub.es

The Scintillator Pad Detector (SPD) is part of the electromagnetic calorimeter of the LHCb experiment. It is meant to separate photons and electrons at level 0 of the trigger. Particles generated in the electromagnetic shower in the preshower and in the electromagnetic calorimeter (backsplash) can affect the efficiencies in $e / \gamma$ separation. Both experimental and Monte Carlo studies of this effect are presented here.

International Europhysics Conference on High Energy Physics

July 21st - 27th 2005

Lisboa, Portugal

${ }^{*}$ Speaker. 


\begin{tabular}{|c|c|c|c|c|c|}
\hline $\begin{array}{c}\text { Beam energy } \\
(\mathrm{GeV})\end{array}$ & $\begin{array}{c}\mathrm{I}+\mathrm{B}+\mathrm{P} \\
(\%)\end{array}$ & $\begin{array}{c}\mathrm{I}+\mathrm{B} \\
(\%)\end{array}$ & $\begin{array}{c}\mathrm{B} \\
(\%)\end{array}$ & $\begin{array}{c}\text { Monte Carlo I } \\
(\%)\end{array}$ & $\begin{array}{c}\text { Monte Carlo B } \\
(\%)\end{array}$ \\
\hline 20 & $5.34 \pm 0.13$ & $<3.9$ & $<1.2$ & $1.26 \pm 0.06$ & $0.56 \pm 0.04$ \\
50 & $5.99 \pm 0.14$ & $<4.8$ & $<1.7$ & $1.39 \pm 0.06$ & $0.89 \pm 0.05$ \\
\hline
\end{tabular}

Table 1: Fraction of photons, of different energies, with a deposited energy above the 0.70 MIP threshold for all selected events (inteaction, I, backsplash, B and pairs $e^{+} e^{-}, \mathrm{P}$, contributions); after removing an (under)estimation of the contribution from electron pairs produced in air (I+B); and after additionally subtracting an (under)estimation of the contribution from photon interactions in the scintillator (B). Monte Carlo estimated interaction and backsplash contributions, two last columns.

\section{Introduction.}

The LHCb experiment $[1,2]$ is designed to study B meson physics in the LHC proton-proton collider at CERN. The electromagnetic calorimeter (ECAL) and its preshower (PS) cannot, by themselves, distinguish between high-pt photons and electrons at the level-0 of the LHCb trigger system. The Scintillator Pad Detector (SPD) [3] is a scintillator plane in front of the calorimeter system, meant to perform the $e / \gamma$ separation at this very first level of triggering.

Charged particles crossing the scintillator produce ionisation, while neutrals contribute little. However, there are effects that can made the photon deposit energy in the SPD, such as photon conversion before the SPD, interaction within the scintillator material and interaction of particles travelling backwards from the PS or ECAL electromagnetic showers, this last called backsplash. Therefore a cut of 0.7 MIP (Minimum Ionizing Particle) on the deposited energy is performed, minimising these effects and without loss in electron identification efficiency.

\section{Experimental results.}

Two experimental measurements were held at CERN. The first one, in 1999, allowed the study of $180^{\circ}$ backsplash, i.e. at the same cell it impinges, with photons as incident particle [4]. The photons were obtained from an electron beam, X7 beam [5], when crossing a lead plate. Then, electrons are deflected by a magnetic field and those from a given energy are selected with the assumption that the rest of the energy is taken by a photon emited at the radiator.

For the beam energies used, photons of 14-18 and 44-48 GeV incide the SPD, PS and ECAL system. The deposited energy at a SPD cell has the contributions of interaction with the scintillator, interaction of backsplash particles and electron pairs produced between veto and SPD. The electron-positron pair and interaction contributions can be substracted, so finally the fraction of misidentified photons in each case are in table 1, which are compatible with the ones obtained from GEANT3 [6] simulation, shown in the two last columns of the table.

The second test, in 2003, aimed to measure the backsplash produced by other particles crossing near cells, called lateral backsplash. It was used electrons and pions, to study both electromagnetic and hadronic cases [7]. The same X7 beam was used at CERN, providing electrons (20 and $50 \mathrm{GeV}$ ) and pions $(50 \mathrm{GeV})$. In front of some ECAL modules and a lead plate of $2 \mathrm{~cm}$ width, a 


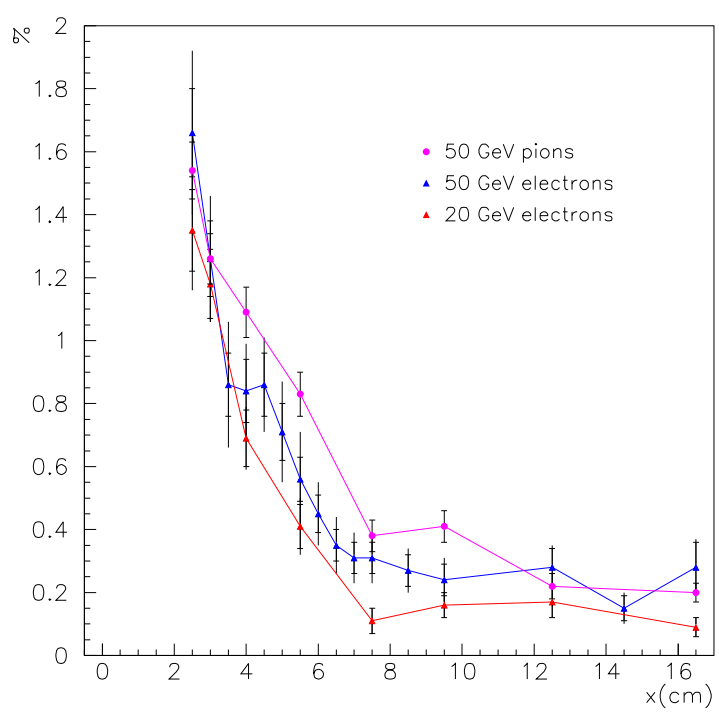

Figure 1: Fraction of particles that deposit an energy greater than 0.7 MIP in the $4 \times 4$ cell (for the selected events) as a function of the $\mathrm{x}$ distance to the beam. Statistical and systematic uncertainty quoted (the lines on the error bars correspond to the statistical one).

$4 \times 4 \times 1 \mathrm{~cm}^{3}$ scintillator is placed in the roll of a SPD cell. It is mobable along an axis perpendicular to the beam, allowing the study of backsplash as a function of the distance to the beam.

At Fig. 1 it can be seen the fraction of particles that deposit an energy higher than 0.7 MIP in the SPD cell due to backsplash, as a function of the distance with the beam. It can be concluded that the effect produced by $50 \mathrm{GeV}$ electrons is approximately $50 \%$ higher than the one produced by $20 \mathrm{GeV}$ electrons and of the same order than the one produced by $50 \mathrm{GeV}$ pions. Monte Carlo simulation is compatible with experimental data in case of electrons, using EGS4 [8] and GEANT3 packages, but simulations for pions using GEANT3 show a higher contribution of backsplash that the one measured.

\section{Occupancies and photon identification efficiency.}

Once seen that Monte Carlo simulations for electrons and photons describe correctly the backsplash contribution to the SPD deposited energy, they can be used to estimate occupancies and photon identification efficiency, among others. For electrons and photons it is used EGS4, simulating the SPD, PS and ECAL system with distances and widths as in the real experiment. For hadrons it is used GEANT3, with only SPD simulated, and backsplash contribution will be estimated using from the electron one, for high energies.

With the reconstruction software of the experiment, Brunel [9], for Monte Carlo minimum bias events (proton-proton collision), it can be obtained the average number of each kind of particles and energy distributions when inciding the SPD. This information and the previous simulations allow to make the estimations of the fraction of events with a deposited energy above the SPD threshold 
and deposited energies for a minimum bias LHCb event, shown at table 2. For the hottest SPD cell, where more particles incide, less than $2.2 \%$ of photons will be detected as a charged particle.

\begin{tabular}{|c|c|c|}
\hline SPD region & $E_{\text {dep }}(\mathrm{keV})$ & $E_{\text {dep }}>0.7$ MIP (\%) \\
\hline Inner & 80.9 & 1.5 \\
\hline Middle & 31.0 & 0.8 \\
\hline Outer & 29.6 & 0.7 \\
\hline
\end{tabular}

Table 2: Average deposited energy and fraction of hits, in a cell per minimum bias event, by the forward particles taking into account backsplash.

\section{Conclusions.}

From experimental measurements, Monte Carlo simulations of backsplash effects are validated and these are used to estimate occupancies and the probability of photon misidentification in a minimum bias event. For the geometry of the LHCb calorimeters, backsplash does not introduce significantly bigger inefficiencies for the e $/ \gamma$ separation than does the interaction of the photon with the SPD material itself, while the increase on occupancy due to backsplash is lower than the $5 \%$ relative.

\section{References}

[1] LHCb Collaboration, S. Amato et al., A Large Hadron Collider Beauty experiment for precision measurements of CP-violation and rare decays. The LHCb Technical Proposal. CERN/LHCC 98-4.

[2] LHCb Collaboration, R. Antunes et al., LHCb Reoptimized Detector Design and Performance. CERN/LHCC 2003-030.

[3] LHCb Collaboration, S. Amato et al., LHCb Calorimeters Technical Design Report. CERN/LHCC/2000-0036.

[4] D. Peralta et al., Results of a tagged photon test beam for the Scintillator Pad Detector. NIM A 484 (2001) 333-341.

[5] Introduction to the $X 7$ beam, Lau Gatignon. http://gatignon.home.cern.ch/gatignon/X7manual.html

[6] Detector Description and Simulation Tool. CERN Program Library Long Writeup W5013, 1993.

[7] M. Calvo et al., Backsplash testbeam results for the SPD subdetector of LHCb. Nucl. Instr. and Methods A 546 (2005) 438-447.

[8] http://www.slac.standford.edu/egs/

[9] Brunel - The LHCb reconstruction program. http://cern.ch/lhcb-comp/Reconstruction 